\title{
Ueber die Steinersche Fläche vierten Grades.
}

(Von Herrn Schröter zu Breslau.)

(Abgedruckt aus dem Monatsbericht der Berliner Akademie vom 26. Nov. 1863.)

Die zu Herrn Kummers Abhandlung: ,ïber die Flächen vierten Grades, auf welchen Schaaren von Kegelschnitten liegen" *) hinzugefügten Bemerkungen des Herrn Weierstrass hinsichtlich der synthetischen Betrachtungen, welche Steiner zu jener merkwürdigen Fläche vierten Grades geführt haben, deren sämmtliche Berührungsebenen Kegelschnittpaare ausschneiden - wurden mir eine Veranlassung, den dort angedeuteten Weg weiter zu verfolgen und ich erlaube mir, die Ergebnisse dieser Untersuchung in dem Folgenden mitzutheilen.

1. Wenn man in der Ebene eines Kegelschnitts zu einem beliebigen Punkte $x$ die Polare $X$ bestimmt, auf derselben einen Punkt $y$ nimmt und dessen Polare $\boldsymbol{Y}$ bestimmt, so ist der Schnittpunkt $z$ von $X, Y$ der Pol der Geraden $Z$, welche $x, y$ verbindet. Solche drei Punkte heissen ein Tripel conjugirter Punkte in Bezug auf den Kegelschnitt. Hält man $x$ fest und verändert $y$ auf $X$, so verändert sich auch $z$; die Punktenpaare $y, z$ bilden ein Punktsystem (Involution) auf $\boldsymbol{X}$, folglich die Strahlenpaare $\boldsymbol{Y}, \boldsymbol{Z}$ ein, mit jenem perspectivisches Strahlsystem. Lässt man den Punkt $x$ die ganze Ebene durchwandern, so durchstreift auch $X$ die ganze Ebene; sie durchlaufen zwei auf einander liegende Gebilde von doppelter Unendlichkeit: ein Punktenfeld und Strahlenfeld, die in reciproker Beziehung zu einander stehen und in besonderer Weise auf einander liegen. Ein solches Doppelgebilde heisst ein ebenes. Polarsystem und kann auch aufgefasst werden als eine Unendlichkeit von Tripeln in Bezug auf den Kegelschnitt. Dieser erscheint in der Ebene des Polarsystems nur als der Ort solcher besonderen Punkte $x$, deren Polarén $X$ durch sie selbst hindurchgehen.

Nimmt man von irgend einem Punkte $O$ des Raumes aus die Perspective eines ebenen Polarsystems, so erhält man in $O$ ein Doppelgebilde von zwei in gewisser Weise concentrisch liegenden, reciproken Bündeln, einem Strahlenbündel und einem Ebenenbündel; ein solches Doppelgebilde

*) Monatsbericht der Berliner Akademie vom 16. Juli 1863. 
heisse ein Polarbündel und kann auch aufgefasst werden als aus unendlich vielen Dreikanten (Tripeln conjugirter Strahlen) bestehend, die alle in dem gemeinsamen Mittelpunkte des Polarbündels zusammenlaufen und durch je ein Tripel des ebenen Polarsystems gehen.

Wenn man von allen Punkten $x$ einer Ebene $\boldsymbol{E}$ die Polarebenen in Bezug auf eine beliebige Oberfläche zweiter Ordnung bestimmt, die sämmtlich durch einen Punkt 0 , den Pol von $E$ laufen, und wenn die Polarebene von $\boldsymbol{x}$ in $\boldsymbol{X}$ die Ebene $E$ schneidet, so' bilden $x, X$ ein ebenes Polarsystem, der Strahl $O x$ und die Polarebene zu $x$ ein mit demselben perspectivisches Polarbündel. Das ebene Polarsystem ist auch hinsichtlich des Kegelschnitts, in welchem $\boldsymbol{E}$ von der Fläche zweiter Ordnung geschnitten wird, ein ebenes Polarsystem; es hört aber nicht auf, reell zu bestehen, wenn der ganze Kegelschnitt imaginär ist. Jede Ebene im Raume enthält daher in Bezug auf die Oberfäche zweiter Ordnung ein bestimmtes ihr zugehöriges Polarsystem und jeder Punkt im Raume ist Mittelpunkt eines bestimmten ihm zugehörigen Polarbündels; beide liegen perspectivisch, wenn Punkt und Ebene Pol und Polarebene sind. Wenn insbesondere die Ebene $E$ die unendlich-entfernte ist, so ist der Pol derselben in Bezug auf die Fläche zweiter Ordnung der Mittelpunkt der letzteren; das Polarbündel, welches ihm zugehört, als eine Unendlichkeit von Dreikanten aufgefasst, ist das System der conjugirten Durchmesser der Fläche.

2. Der a. a. 0. von Herrn Weierstrass mitgetheilte erste Steinersche Satz lautet in dem angegebenen Sinne erweitert, folgendermaassen:

Wenn man den Mittelpunkt eines Polarbündels in eine Fläche zweiter Ordnung verlegt, so geht die Verbindungsebene solcher drei Punkte, in welchen jedes Tripel conjugirter. Strahlen dieselbe trifft, durch einen festen Punkt, und dies ist die unmittelbare Ausdehnung des bekannten Satzes in der Ebene:

Wenn man den Mittelpunkt eines Strahlsystems in die Peripherie eines Kegelschnitts verlegt, so geht die Verbindungslinie solcher zwei Punkte, in welchen je zwei conjugirte Strahlen des Strahlsystems den Kegelschnitt treffen, durch einen festen Punkt.

Nehmen wir zum Beweise einen beliebigen Strahl $a$ und die conjugirte Ebene $\boldsymbol{A}$ des Polarbündels $\boldsymbol{O}$ und treffe ersterer die Fläche zweiter Ordnung $F^{(2)}$ in $\mathfrak{a}$ und letztere in dem Kegelschnitt $\mathfrak{A}^{(2)}$, so werden alle Strahlenpaare $b, c$ in der Ebene $A$, welche mit $a$ zusammen ein Tripel conjugirter Strahlen des Polarbündels sind, ein Strahlsystem bilden und daher den Kegelschnitt $\mathfrak{A}^{(2)}$ in Punktenpaaren treffen, deren Verbindungslinien durch einen festen 
Punkt $\alpha$ laufen. Die Verbindungsebenen der Durchschnittspunkte von $F^{(2)}$ mit solchen drei Tripelstrahlen, deren einer $a$ fest bleibt, während die beiden anderen $b, c$ in der Ebene $A$ variiren, laufen daher durch eine feste Gerade $\mathfrak{a} \alpha$. Denken wir uns die Tangentialebene $T$ im Punkte $O$ an $F^{(2)}$ und den derselben conjugirten Strahl $t$ im Polarbündel bestimmt, so muss letzterer die Gerade $a \alpha$ treffen; denn da die Schnittlinie $s$ von $T$ und $A$ Tangente an $\mathfrak{2}^{(2)}$ ist und der dritte Tripelstrahl $\sigma$ zu $a$ und $s$ erhalten wird, indem wir eine Ebene durch $a, t$ legen, die $A$ in $\sigma$ schneidet, so muss die Verbindungslinie der Schnittpunkte des Paares $s$, $\sigma$ mit $\mathfrak{A}^{(2)} \sigma$ selbst sein also durch $\alpha$ gehen, d. h. die durch $a$ und $t$ gelegte Ebene enthält $\alpha$, also $\mathfrak{a} \alpha$ wird von $t$ getroffen. Dasselbe gilt von allen auf gleiche Weise wie $a \alpha$ construirten Geraden. Nehmen wir nun im Polarbündel ein beliebiges zweites Paar $b, B$, welche in dem Punkt $\mathfrak{b}$ und dem Kegelschnitt $\mathfrak{B}^{(2)}$ der Fläche $F^{(2)}$ begegnen, und sei $\beta$ der Punkt in der Ebene $B$, welcher in derselben Weise wie $\alpha$ in $A$ erhalten wird, so müssen nicht nur $\mathfrak{\alpha} \alpha$ und $\mathfrak{b} \beta$ von $t$ getroffen werden, sondern auch sich selbst treffen. Die Ebenen $A, B$ schneiden sich nämlich in einem Strahle $c$, dessen conjugirte Ebene $C$ die durch $a, b$ gelegte ist; der conjugirte Strahl zur Ebene $(a, c)$ ist die Schnittlinie $(A, C)$ und treffe $F^{(2)}$ in $\mathfrak{a}^{\prime}$; der conjugirte Strahl zu $(b, c)$ ist die Schnittlinie $(B, C)$ und treffe $F^{(2)}$ in $\mathfrak{b}^{\prime}$; die Linien $\mathfrak{a a}^{\prime}$ und $\mathfrak{b b}^{\prime}$, welche in der Ebene $C$ liegen, treffen sich in $\gamma$, und $c \gamma$ ist offenbar wiederum eine Linie der Art, wie $a \alpha$ und $\mathfrak{b} \beta$, muss also auch der Geraden $t$ begegnen. Da nun die drei nach 'caa' hingehenden Strahlen ein Tripel conjugirter Strahlen des Polarbündels sind, so muss $\alpha$ auf ca' liegen, also $\alpha \mathfrak{c a}^{\prime} \mathfrak{a}$ in einer Ebene und da $\gamma$ auf $\mathfrak{a} \mathfrak{a}^{\prime}$ liegt, $a \alpha$ und $c \gamma$ in einer Ebene; aus gleichem Grunde auch $\mathfrak{b} \beta$ und $c \gamma$ in einer Ebene; wenn nun $a \alpha$ und $\mathfrak{b} / \beta$ sich nicht träfen, so müsste die feste Gerade $t$, weil sie alle drei Geraden $\mathfrak{a} \alpha, \mathfrak{b} \beta, \mathfrak{c} \gamma$ treffen muss, entweder in der durch $\mathfrak{a} \alpha, \mathfrak{c} \gamma$ oder in der durch $\mathfrak{b} / \beta, \mathfrak{c} \gamma$ bestimmten Ebene liegen und durch den Schnittpunkt der jedesmaligen anderen Geraden gehen. Die Paare $a, A$ und $b, B$ sind aber ganz willkührlich gewählt; verändern wir daher das eine, während wir das andere festhalten, so müsste $t$ entweder mit $\mathfrak{a} \alpha$ oder mit $\mathfrak{b} \beta$ zusammenfallen, was widersinnig ist. Es müssen also $\mathfrak{a} \alpha$ und $\mathfrak{b} \beta$ selbst sich treffen und da $t$ im Allgemeinen nicht mit beiden zugleich in derselben Ebene liegen wird, so muss $t$ durch den Schnittpunkt beider hindurchgehen. Verändern wir nun das eine Paar $b, B$ beliebig im Polarbündel, so müssen alle Geraden $\mathfrak{b} \beta$ durch einen festen Punkt, den Schnittpunkt von $t$ mit $a \alpha$, gehen, also überhaupt die 
Verbindungsebenen der Durchschnittspunkte von $F^{(2)}$ mit je drei Tripelstrahlen des Polarbündels durch einen festen Punkt laufen, w. z. b. w.

3. Wenn in der Ebene ein Büschel von Kegelschnitten $K^{(2)}$, welche durch dieselben vier (reellen oder imaginären) Schnittpunkte von zweien derselben hindurchgehen, gegeben ist, so bestimmt jeder dieser Kegelschnitte ein ebenes Polarsystem (1.) und wenn wir eine Fläche $F^{(2)}$ mit einem auf ihr befindlichen Punkt $O$ beliebig annehmen, so liefert nach dem vorigen Satze (2.) jedes Polarsystem einen gewissen Punkt $s$ im Raume als Durchschnittspunkt solcher Ebenen, welche je drei Schnittpunkte der Fläche $F^{(2)}$ mit drei von $O$ nach den Tripeln des Polarsystems hin gezogenen Strahlen verbinden. Da also zu jedem Kegelschnitte $K^{(2)}$ des Büschels ein bestimmter Punkt $s$ im Raume zugehört, so können wir nach dem Orte der Punkte $s$ für sämmtliche Kegelschnitte des Büschels fragen. Bekanntlich haben alle Kegelschnitte eines Büschels ein gemeinschaftliches Tripel (Diagonalpunkte des von den vier Mittelpunkten des Büschels gebildeten vollständigen Vierecks); also wird diejenige Ebene, welche die Durchschnittspunkte von $F^{(2)}$ mit den drei von $O$ nach dem gemeinschaftlichen Tripel gezogenen Strahlen verbindet, alle Punkte $s$ enthalten; um nun einen Punkt $s$ zu erhalten, ist es nur noch nöthig, die Tangentialebene in $O$ an $F^{(2)}$ zu legen, welche die Ebene des Kegelschnittbüschels in einer Geraden $\mathfrak{R}$ schneiden wird, alsdann den Pol von $\mathbb{R}$ in Bezug auf einen Kegelschnitt des Büschels mit $O$ zu verbinden und den Schnittpunkt $s$ dieser Verbindungslinie mit der vorhin ermittelten Ebene zu fixiren. Die sämmtlichen Pole von $\mathfrak{Z}$ in Bezug auf alle Kegelschnitte des Büschels liegen aber bekanntlich auf einem bestimmten Kegelschnitt $\Re$, der durch das gemeinschaftliche Tripel des Büschels hindurchgeht und der gesuchte Ort der Punkte $s$ ist, daher der Durchschnitt der vorhin ermittelten Ebene mit den von $O$ nach dem Kegelschnitt $\Re$ gezogenen Kegelstrahlen, folglich ein Kegelschnitt. Dies kommt mit dem zweiten von Herrn Weierstrass a. a. 0. mitgetheilten Satze überein, denn ein ebenes Polarsystem wird sowohl durch einen Kegelschnitt bestimmt, als auch durch eine beliebige Fläche zweiten Grades, die jenen Kegelschnitt enthält (1.); zu einer solchen Gruppe von Polarsystemen, wie sie zuletzt betrachtet ist, hätten wir anstatt mittelst eines Kegelschnittbüschels auch mittelst eines Büschels von Flächen zweiter Ordnung gelangen können, welche durch dieselbe Raumcurve vierter Ordnung (Schnittcurve zweier bestimmter Flächen) gehen. Die erstere Auffassung scheint aber bequemer zu sein, weil dabei nur in der Ebene zu operiren ist und wir werden sie daher auch im Fol- 
genden festhalten, da die Allgemeingültigkeit des Resultates nicht beeinträchtigt wird, obschon die Betrachtung nur für den Fall der Realität von Theilen der Figur, welche auch imaginär' werden können, durchgeführt ist.

4. Sind drei beliebige Kegelschnitte $A, B, C$ in der Ebene gegeben, so ist der Ort solcher Punkte, deren drei Polaren in Bezug auf jene sich in einem Punkte treffen eine allgemeine Curve dritten Grades, welche durch die gemeinschaftlichen Tripel der paarweise zusammengenommenen Kegelschnitte $(A, B)(B, C)(C, A)$ hindurchgeht und durch diese 9 Punkte vollständig bestimmt wird $\left.{ }^{*}\right)$.

Seien $\alpha \alpha^{\prime} \alpha^{\prime \prime}, \beta \beta^{\prime} \beta^{\prime \prime}, \gamma \gamma^{\prime} \gamma^{\prime \prime}$ die gemeinschaftlichen Tripel der Kegelschnittpaare $(B, C),(C, A),(A, B)$, sei $G$ eine beliebige Gerade, deren Pole in Bezug auf $A, B, C$ respective $\mathfrak{a}, \mathfrak{b}, \mathfrak{c}$ heissen mögen und bewegen wir auf $G$ einen veränderlichen Punkt $P$, dessen Polaren in Bezug auf $A, B, C$ respective $a, b, c$ seien, so dreht sich $a$ um $a, b$ um $\mathfrak{b}, c$ um $\mathfrak{c}$ und $a, b, c$ beschreiben drei mit der von $\boldsymbol{P}$ durchlaufenen Punktreihe projectivische Strahlbüschel; der Ort des Schnittpunktes $(b, c)$ ist daher ein Kegelschnitt, welcher durch $\alpha \alpha^{\prime} \alpha^{\prime \prime} \mathfrak{b c}$, der Ort des Schnittpunktes $(c, a)$ ein zweiter Kegelschnitt, welcher durch $\beta \beta^{\prime} \beta^{\prime \prime} \mathfrak{c a}$ geht; beide Kegelschnitte haben den Punkt $c$ mithin noch drei andere Punkte $Q Q^{\prime} Q^{\prime \prime}$ gemein, welche die verlangte Eigenschaft besitzen, dass für drei gewisse Punkte $P P^{\prime} P^{\prime \prime}$ der Geraden $G$ die drei Polaren in Bezug auf die gegebenen Kegelschnitte sich in je einem der Punkte $Q$, $Q^{\prime}, Q^{\prime \prime}$ treffen. Ein solches Paar $P, Q$ heissen conjugirte Punkte, weil auch die Polaren von $Q$ in Bezug auf die drei gegebenen Kegelschnitte durch $P$ laufen. Die conjugirten Punkte $P P^{\prime} P^{\prime \prime}$ sind auf der Geraden $G$ leicht zu bestimmen, sobald man $Q Q^{\prime} Q^{\prime \prime}$ gefunden hat. Sobald nämlich $P, Q$ ein Paar conjugirter Punkte in Bezug auf den Kegelschnitt $A$ sind (d. h. zwei solche Punkte, dass die Polare von $P$ durch $Q$ und also auch durch die Polare von $Q$ durch $P$ geht) und $P^{\prime} Q^{\prime}$ ein zweites Paar conjugirter Punkte in Bezug auf denselben Kegelschnitt, so ist leicht $\mathrm{zu}$ erweisen, dass die beiden Schnittpunkte der Verbindungslinien $\left(P P^{\prime}, Q Q^{\prime}\right)$ und $\left(P Q^{\prime}, Q P^{\prime}\right)$ nothwendig ein drittes Paar conjugirter Punkte in Bezug auf denselben Kegelschnitt sein müssen. Da nun diese Eigenschaft für unsere Punkte $P, Q$ und $P^{\prime} Q^{\prime}$ in Bezug auf alle drei Kegelschnitte $A, B, C$ stattindet, so gilt sie auch für das dritte Paar.

*) Dieser Satz ist von Herrn Hesse im XXVIII. Bande dieses Journals Seite 105 angegeben und analytisch bewiesen. 
Es giebt aber auf der Geraden $P P^{\prime}$ oder $\boldsymbol{G}$ nur einen einzigen dritten Punkt $P^{\prime \prime}$, dessen drei Polaren sich in demselben Punkte $Q^{\prime \prime}$ treffen, folglich muss $P^{\prime \prime}$ der Schnittpunkt sein, in welchem $Q Q^{\prime}$ die Gerade $G$ trift und $Q^{\prime \prime}$ der Schnittpunkt von $P Q^{\prime}$ und $Q P^{\prime}$ d. h. $P, P^{\prime}, P^{\prime \prime}$ sind diejenigen drei Punkte, in welchen die Seiten des Dreiecks $Q Q^{\prime} Q^{\prime \prime}$ der Geraden $G$ begegnen und zwar liegen $Q Q^{\prime} P^{\prime \prime}, Q^{\prime} Q^{\prime \prime} P, Q^{\prime \prime} Q P^{\prime}$ in je einer Geraden oder die sechs Punkte $P P^{\prime} P^{\prime \prime} Q Q^{\prime} Q^{\prime \prime}$ sind die sechs Ecken eines vollständigen Vierseits.

Da auf der beliebigen Geraden $G$ nur drei Punkte der verlangten Eigenschaft existiren, so ist der Ort sämmtlicher Punkte $P, Q$ eine Curve dritten Grades, welche sich auch in folgender Weise construiren lässt: denken wir uns die Gerade $G$ um einen beliebigen festen Punkt $O$ gedreht, so verändern sich mit ihr die Punkte $Q Q^{\prime} Q^{\prime \prime}$; von den beiden Kegelschnitten, deren jedesmalige Schnittpunkte sie sind, geht der erste durch die drei festen Punkte $\alpha \alpha^{\prime} \alpha^{\prime \prime}$, während die beiden Punkte $\mathfrak{b}$ und $c$ auf den Polaren von $O$ in Bezug auf $B$ und $C$ zwei gerade Punktreihen durchlaufen; da der Schnittpunkt $\alpha_{0}$ dieser beiden Polaren nothwendig auch auf dem Kegelschnitte liegt, so beschreibt dieser bei seiner Veränderung ein Kegelschnittbüschel von vier festen Punkten $\alpha \alpha^{\prime} \alpha^{\prime \prime} \alpha_{0}$; ebenso beschreibt der zweite Kegelschnitt ein Büschel von vier Punkten $\beta \beta^{\prime} \beta^{\prime \prime} \beta_{0}$, wenn $\beta_{0}$ den Schnittpunkt der Polaren von $O$ in Bezug auf die Kegelschnitte $C$ und $A$ bezeichnet; der veränderliche Punkt c, den beide Kegelschnitte gemein haben, liegt auf der festen Verbindungslinie $\alpha_{0} \beta_{0}$ der Polare von $O$ in Bezug auf $C$. Hieraus folgt, dass die beiden Büschel projectivisch sind, also der Ort ihrer drei anderen jedesmaligen Schnittpunkte $Q Q^{\prime} Q^{\prime \prime}$ eine allgemeine Curve dritten Grades ist, welche durch $\alpha \alpha^{\prime} \alpha^{\prime \prime} \beta \beta^{\prime} \beta^{\prime \prime}$, aber nicht durch $\alpha_{0} \beta_{0}$ geht. Die Punkte $\gamma \gamma^{\prime} \gamma^{\prime \prime}$ des gemeinschaftlichen Tripels von $A$ und $B$ liegen auch auf dieser Curve dritten Grades, denn die Polaren von $\gamma$ in Bezug auf $A$ und $B$ fallen zusammen in die Linie $\gamma^{\prime} \gamma^{\prime \prime}$, folglich schneiden sich alle drei Polaren von $\gamma$ in Bezug auf $A, B, C$ in einem Punkte. Durch die neun Punkte $\alpha \alpha^{\prime} \alpha^{\prime \prime} \beta \beta^{\prime} \beta^{\prime \prime} \gamma \gamma^{\prime} \gamma^{\prime \prime}$ ist die Curve dritten Grades vollständig bestimmt $d$. h. es können keine zwei Curven dritten Grades durch dieselben gehen; sonst müsste nämlich ein ganzes Büschel Curven dritten Grades hindurchgehen und da $\alpha \alpha^{\prime} \alpha^{\prime \prime}\left(\beta^{\prime} \beta^{\prime} \beta^{\prime \prime}\right.$ sechs Punkte eines Kegelschnitts sind, weil zwei Tripel desselben Kegelschnitts $C$ immer auf einem Kegelschnitt liegen, so müssten $\gamma \gamma^{\prime} \gamma^{\prime \prime}$ auf einer Geraden liegen; dies ist aber unmöglich, weil drei Tripelpunkte im Allgemeinen nicht in einer Geraden liegen; also kann nur eine Curve dritten Grades durch jene neun Punkte gehen. 
Aus dem Vorigen folgt zugleich, dass ein dritter durch die Punkte $\gamma \gamma^{\prime} \gamma^{\prime \prime} \mathfrak{a}$ gelegter Kegelschnitt die Punkte $Q Q^{\prime} Q^{\prime \prime}$ enthalten muss; und da dieser Kegelschnitt auch als der Ort der Pole der Geraden $G$ in Bezug auf sämmtliche Kegelschnitte des durch $\boldsymbol{A}$ und $\boldsymbol{B}$ bestimmten Büschels angesehen werden kann, so folgt ein Satz, von dem wir später Gebrauch machen:

Drei beliebige Kegelschnitte $A, B, C$ bestimmen drei Büschel $(B, C)$, $(C, A),(A, B)$; die Pole einer Geraden $G$ in Bezug auf alle Kegelschnitte eines Büschels liegen auf einem Kegelschnitt; die auf diese Weise für die drei Büschel erhaltenen Kegelschnitte laufen durch dieselben drei Punkte.

5. Die drei Kegelschnitte $A, B, C$ geben zu zweien combinirt drei Kegelschnittbüscheln $(B, C),(C, A),(A, B)$ ihre Entstehung; die vorhin ermittelten Punkte $Q Q^{\prime} Q^{\prime \prime}$ als die conjugirten zu gewissen drei auf einer gegebenen Geraden $G$ liegenden $P P^{\prime} P^{\prime \prime}$ stehen noch in folgender merkwürdigen Beziehung zu den Büscheln. Die Polaren von $Q$ in Bezug auf alle Kegelschnitte des Büschels $(B, C)$ gehen durch $P$, folglich wird die Gerade $P Q^{\prime} Q^{\prime \prime}$ die Polare von $Q$ in Bezug auf einen besondern Kegelschnitt $\mathfrak{A}$ des Büschels $(B, C)$ sein und ebenfalls in Bezug auf einen besondern Kegelschnitt $\mathfrak{B}$ des Büschels $(C, A)$ und einen besondern $\subseteq$ des Büschels $(A, B)$. Die Kegelschnitte $\mathfrak{A} \mathfrak{B} \subseteq$ sind vollständig und eindeutig durch diese Bedingung bestimmt. Für die beiden Kegelschnitte $\mathfrak{A}$ und $\mathfrak{B}$ muss daher $Q$ ein Punkt des gemeinsamen Tripels sein und $Q^{\prime} Q^{\prime \prime}$ seine Polare; die Polare von $Q^{\prime}$ in Bezug auf $\mathfrak{A}$ und $\mathfrak{B}$ muss daher durch $Q$ gehen, zugleich aber auch durch $P^{\prime}$, den conjugirten Punkt zu $Q^{\prime}$ in Bezug auf alle drei Kegelschnitte $A, B, C$ also auch in Bezug auf $\mathfrak{A}$ und $\mathfrak{B}$; da nun $P^{\prime} Q Q^{\prime \prime}$ in einer Geraden liegen, so ist $Q Q^{\prime \prime}$ die Polare von $Q^{\prime}$ für beide Kegelschnitte $\mathfrak{A}$ und $\mathfrak{B}$, folglich $Q Q^{\prime} Q^{\prime \prime}$ das gemeinsame Tripel der Kegelschnitte $\mathfrak{A}$ und $\mathfrak{B}$ und in gleicher Weise der Kegelschnitte $\mathfrak{B}$ und $\mathfrak{C}$; diese drei neuen Kegelschnitte $\mathfrak{A} \mathfrak{B} \mathfrak{C}$ müssen daher demselben Büschel angehören, weil zwei Kegelschnitte immer nur ein gemeinschaftliches Tripel haben können. Da nun umgekehrt leicht einzusehen ist, dass zwei beliebige den Büscheln $(B, C)$ und $(C, A)$ entnommene Kegelschnitte $\mathfrak{Y}$ und $\mathfrak{B}$ ein gemeinsames Tripel $Q Q^{\prime} Q^{\prime \prime}$ haben, welches auf der in (4.) ermittelten Curve dritlen Grades liegen muss, so folgt zunächst ein Satz, von welchem später Gebrauch gemacht wird:

Sind B,C, $\mathfrak{A}$ drei Kegelschnitte eines Büschels und $C, A, \mathfrak{B}$ drei Kegelschnitte eines zweiten Büschels, welches den Kegelschnitt $C$ mit dem vorigen gemein hat, so giebt es einen Kegelschnitt $\mathfrak{E}$, der sowohl in dem durch 
$A$ und $B$ bestimmten Büschel, als auch in dem durch $\mathfrak{A}$ und $\mathfrak{B}$ bestimmten Büschel liegt, $d$. . . die acht Mittelpunkte der Bilschel $(A, B)$ und $(\mathfrak{A}, \mathfrak{B})$ liegen auf einem Kegelschnitt $\mathfrak{E}$, und weiter, da $\mathfrak{A}$ und $\mathfrak{B}$ zwei willkührlich aus den drei Büscheln $(B, C),(C, A),(A, B)$ entnommene Kegelschnitte bedeuten, deren gemeinschaftliches Tripel $Q Q^{\prime} Q^{\prime \prime}$ ist, dass der Ort dieser unendlichen Mannigfaltigkeit von Tripeln ein und dieselbe Curve dritten Grades ist, welche bereits (4.) als der Ort der in Bezug auf die drei Kegelschnitte $A, B, C$ gleichzeitig conjugirten Punktpaare $P, Q$ gefunden wurde. Diese Curve heisse daher kurzweg Tripelcurve und solche drei Punkte, wie $Q Q^{\prime} Q^{\prime \prime}$ ein Tripel'der Tripelcurve. Also:

Ein Tripel $Q Q^{\prime} Q^{\prime \prime}$ der Tripelcurve liegt immer so auf derselben, dass die drei ihnen conjugirten Punkte $P P^{\prime} P^{\prime \prime}$ auf einer Geraden sich befinden und die jedesmaligen dritten Schnittpunkte der Dreiecksseiten des Tripels $Q Q^{\prime} Q^{\prime \prime}$ mit der Tripelcurve sind *)

und auch umgekehrt. Die sechs Punkte $P P^{\prime} P^{\prime \prime} Q Q^{\prime} Q^{\prime \prime}$ bilden daher zu dreien vier Tripel der Tripelcurve und liegen zu dreien auf vier Geraden:

\begin{tabular}{|c|c|c|c|c|c|c|}
\hline$Q Q^{\prime} Q^{\prime \prime}$ & ein & Tripel & $\boldsymbol{P} \boldsymbol{P}^{\prime} \boldsymbol{P}^{\prime \prime}$ & aul & eine & reraden \\
\hline$Q P^{\prime} P^{\prime \prime}$ & - & - & $P Q^{\prime} Q^{\prime \prime}$ & - & - & - \\
\hline$Q^{\prime} P^{\prime \prime} P$ & - & - & $P^{\prime} Q^{\prime \prime} Q$ & - & - & - \\
\hline$Q^{\prime \prime} P P^{\prime}$ & - & - & $P^{\prime \prime} Q Q^{\prime}$ & - & - & - \\
\hline
\end{tabular}

6. Die drei Kegelschnitte $A, B, C$ geben nicht nur drei Büscheln $(B, C)(C, A)(A, B)$ ihre Entstehung sondern unendlich vielen, indem irgend drei aus letzteren herausgenommene Kegelschnitte wiederum drei Büschel bestimmen u. s. f. Die Totalität der allen diesen Büscheln angehörenden Kegelschnitte heisst ein Kegelschnittnetz; um dieselben anschaulicher zu übersehen, denken wir uns aus dem Büschel $(B, C)$ einen veränderlichen Kegelschnitt $\mathfrak{A}$ entnommen, denselben mit $\boldsymbol{A}$ zur Bildung eines neuen Büschels $(A, \mathfrak{A})$ zusammengestellt und dann die Kegelschnitte des Büschels $(A, \mathfrak{A})$ aufgefasst; indem wir $\mathfrak{A}$ sich verändern lassen, erhalten wir unendlich viele Büschel also eine Schaar-Schaar von Kegelschnitten, welches die sämmtlichen Kegelschnitte des Netzes sind. Selbstverständlich gehören dem Netze auch $A B C$ an und es lässt sich zeigen, dass wenn wir drei beliebige andere Kegelschnitte des Netzes, die nicht demselben Büschel angehören, in der eben angegebenen

*) Vergl. Steiner: Sätze über Curven zweiter und dritter Ordnung in diesem Journal Bd. XXXII, Seite $300 \mathrm{ff}$. 
Weise zur Bildung des Netzes verwenden, keine neuen Kegelschnitte mèhr hervorgehen, sondern nur die früheren, aber in anderer Anordnung zu Büscheln vereinigt. Nehmen wir zunächst einen beliebigen Kegelschnitt $\mathfrak{B}$ aus dem Büschel $(C, A)$ und bilden das veränderliche Büschel $(B, \mathfrak{B})$, so muss, weil $B, C, \mathfrak{U}$ einem Bũschel und $C, A, \mathfrak{B}$ einem zweiten Büschel angehören, welches mit dem ersten den Kegelschnitt $C$ gemein hat, nach dem vorhin (5.) bewiesenen Satze ein Kegelschnitt existiren, der den Büscheln $(A, \mathfrak{A})$ und $(B, \mathfrak{B})$ gemeinschaftlich ist. Verändern wir nun $\mathfrak{A}$, so giebt es in jedem Büschel $(A, \mathfrak{A})$ einen Kegelschnitt, der bei beliebig gewählten $\mathfrak{B}$ dem Büschel $(B, \mathfrak{B})$ angehört und umgekehrt verändern wir $\mathfrak{B}$, so giebt es in jedem Büschel $(B, \mathfrak{B})$ einen Kegelschnitt, der bei beliebig gewähltem $\mathfrak{A}$ dem Büschel $(A, \mathfrak{A})$ angehört; also jeder Kegelschnitt aus dem veränderlichen Büschel $(A, \mathfrak{U})$ ist gleichzeitig in den aus dem veränderlichen Büschel $(B, \mathfrak{B})$ hervorgehenden Kegelschnitten enthalten und umgekehrt, also die Schaar-Schaar von Kegelschnitten ist dieselbe, ob wir $(B, C)$ und $A$, oder $(C, A)$ und $B$, oder $(A, B)$ und $C$ zur Bildung des Netzes verwenden. Nehmen wir zweitens irgend einen Kegelschnitt $D$, der einem bestimmten Büschel $\left(A, \mathfrak{A}_{0}\right)$ der unendlich vielen Büschel $(A, \mathfrak{A})$ angehört, heraus, so liegen einmal $D, A, \mathfrak{A}_{0}$ in einem Büschel, zweitens auch $B ; \mathfrak{A}, \mathfrak{A}_{0}$ in einem Büschel $(B, C)$, also haben nach dem obigen Satze $(A, \mathfrak{X})$ und $(B, D)$ einen Kegelschnitt gemein d. h. alle Büschel $(A, \mathfrak{A})$ können auch bestimmt werden durch Kegelschnitte aus dem Büschel $(B, D)$ und umgekehrt, d. h. $(B, C)$ und $A$, oder $(B, D)$ und $A$ zur Bildung des Netzes verwendet liefern dieselbe Schaar-Schaar von Kegelschnitten. Hieraus ergiebt sich, dass ebenso wie $(A, B)$ und $C$ auch $(A, B)$ und $D$ dieselbe Schaar-Schaar liefert und folglich auch $(B, D)$ und $A$, oder $(B, D)$ und $E$, oder $(D, E)$ und $B$, oder $(D, E)$ und $F$, wenn $D, E, F$ drei beliebige Kegelschnitte des Netzes sind, die nicht demselben Büschel angehören, w. z. b. w. *)

* 7. Sind $P, Q$ gleichzeitig conjugirte Punkte in Bezug auf jeden der drei ursprünglichen Kegelschnitte $A, B, C$, so dass also für jeden die Polare von $P$ durch $Q$ geht und umgekehrt, dann ist es ersichtlich, dass sie in Bezug auf alle Kegelschnitte des Netzes conjugirte Punkte sein werden. Die Tripelcurve ist also immer dieselbe, welche drei Kegelschnitte des Netzes

*) Die Schaar-Schaar Kegelschnitte eines Netzes werden analytisch durch die Gleichung $f+\lambda \varphi+\mu \psi=0$ repräsentirt, wo $f=0, \varphi=0, \psi=0$ die Gleichungen dreier beliebiger Kegelschnitte und $\lambda, \mu$ zwei willkührliche Constanten bedeuten. 
man auch zur Bildung desselben wähle; sie enthält mithin sämmtliche gemeinschaftlichen Tripel irgend zweier Kegelschnitte des Netzes und da sich die letzteren in unendlich mannichfacher Weise zu Büscheln zusammenfassen lassen, so giebt es auch eine unendliche Mannichfaltigkeit von Tripeln der Tripelcurve. Irgend zwei Punkte $Q$ und $Q^{\prime}$, willkührlich auf der Tripelcurve gewählt, können als zwei Punkte des gemeinschaftlichen Tripels eines bestimmten Büschels des Netzes aufgefasst werden und es giebt dann nur ein einziges Büschel, welches zu seinem gemeinschaftlichen Tripel dieses willkührlich gewählte Tripel der Tripelcurve hat; man erhält den dritten zugehörigen Tripelpunkt $Q^{\prime \prime}$, indem man zu $Q$ und $Q^{\prime}$ respective die conjugirten Punkte $P$ und $P^{\prime}$ auf der Tripelcurve aufsucht und den Schnittpunkt von $P Q^{\prime}$ und $Q P^{\prime}$ bestimmt, welcher $Q^{\prime \prime}$ ist. Das diesem Tripel $Q Q^{\prime} Q^{\prime \prime}$ zugehörige Büschel des Netzes erhält man, indem man denjenigen Kegelschnitt $\mathfrak{A}$ des Büschels $(B, C)$ aufsucht, für welchen $Q$ und $P Q^{\prime}$ Pol und Polare sind und ebenso denjenigen bestimmten Kegelschnitt $\mathfrak{B}$ des Büschels $(C, A)$ für welchen dasselbe stattfindet; die beiden Kegelschnitte $\mathfrak{A}$ und $\mathfrak{B}$ bestimmen das gesuchte Büschel, wie aus 5. folgt. Nimmt man nur einen Punkt $Q$ willkührlich auf der Tripelcurve, so giebt es unendlich viele Tripel derselben, welche den Punkt $Q$ enthalten; die Verbindungslinie der jedesmaligen beiden anderen Tripelpunkte läuft durch einen festen Punkt $P$ der Tripelcurve, den conjugirten zu $Q$.

Fassen wir zwei beliebige Tripel $Q Q^{\prime} Q^{\prime \prime}$ und $Q_{1} Q_{1}^{\prime} Q_{1}^{\prime \prime}$ der Tripelcurve auf, so lassen sich nach dem Vorigen die beiden Kegelschnitte $\mathfrak{A}$ und $\mathfrak{B}$ respective aus den Büscheln $(B, C)$ und $(C, A)$ ermitteln, für welche $Q Q^{\prime} Q^{\prime \prime}$ das gemeinschaftliche Tripel ist und gleichfalls aus denselben beiden Büscheln die Kegelschnitte $\mathfrak{A}_{1}$ und $\mathfrak{B}_{1}$, deren gemeinschaftliches Tripel $Q_{1} Q_{1}^{\prime} Q_{1}^{\prime \prime}$ ist; da nun $C, \mathfrak{A}, \mathfrak{A}_{1}$ einem Büschel $(B, C)$ und $C, \mathfrak{B}, \mathfrak{B}_{1}$ einem Büschel $(C, A)$ angehören, und der Kegelschnitt $C$ beiden Büscheln gemeinschaftlich ist, so müssen nach dem obigen Satze (5.) die Büschel $(\mathfrak{A}, \mathfrak{B})$ und $\left(\mathfrak{A}_{1}, \mathfrak{B}_{1}\right)$ einen Kegelschnitt gemein haben. Hieraus folgt:

Zwei beliebige Büschel des Netzes haben immer einen und nur einen Kegelschnitt gemein.

Da in Bezug auf diesen gemeinschaftlichen Kegelschnitt sowohl $Q Q^{\prime} Q^{\prime \prime}$ als auch $Q_{1} Q_{1}^{\prime} Q_{1}^{\prime \prime}$ ein Tripel ist und zwei Tripel eines Kegelschnitts bekanntlich immer selbst auf einem Kegelschnitte liegen, so folgt:

Irgend zwei Tripel der Tripelcurve liegen immer auf einem Kegelschnitt. 
Aber auch umgekehrt wird ein durch die drei Punkte eines Tripels der Tripelcurve willkührlich gelegter Kegelschnitt dieselbe in drei neuen Punkten treffen, von denen nach dem Vorigen zwei als Tripelpunkte eines bestimmten andern Tripels angesehen werden dürfen; der dritte zugehörige Tripelpunkt muss aber sowohl auf dem Kegelschnitt nach dem letzten Satze, als auch auf der Tripelcurve liegen, mithin der sechste Schnittpunkt sein; daher:

Ein beliebiger durch ein Tripel der Tripelcurve gelegter Kegelschnitt trifft dieselbe in drei neuen Punkten, welche wieder ein Tripel der Tripelcurve bilden.

Die Totalität sämmtlicher Tripel der Tripelcurve lässt sich hiernach leicht umfassen durch die Schaar-Schaar von Kegelschnitten, welche durch drei feste Punkte irgend eines Tripels hindurchgehen, und da zu jedem Tripel der Tripelcurve nur ein einziges Büschel des. Netzes gehört, so haben wir auch eine Schaar-Schaar von Büscheln des Netzes. In diesen vertheilen sich aber die Kegelschnitte des Netzes, indem sie sich in verschiedenen Büscheln immer wieder reproduciren, derart, dass sie auch nur eine Schaar-Schaar bilden.

Nennen wir den Ort der Pole einer Geraden in Bezug auf alle Kegelschnitte eines Büschels den Polarkegelschnitt der Geraden, so sagt der am Schlusse von (4.) bewiesene Satz aus, dass die drei Polarkegelschnitte einer Geraden in Bezug auf die drei Büschel $(B, C),(C, A),(A, B)$ sich in denselben drei Punkten treffen und lässt sich jetzt dahin verallgemeinern, dass die Polarkegelschnitte derselben Geraden in Bezug auf sämmtliche Büschel eines Netzes durch drei feste Punkte gehen, welche ein Tripel der Tripelcurve bilden und die conjugirten Punkte zu den drei Schnittpunkten der Geraden mit der Tripelcurve sind.

Denn nehmen wir aus den Büscheln $(B, C)$ und $(C, A)$ zwei beliebige Kegelschnitte $\mathfrak{A}$ und $\mathfrak{B}$ heraus, so bestimmen $(C, \mathfrak{A})$ dasselbe Büschel wie $(B, C)$ und $(C, \mathfrak{B})$ dasselbe wie $(C, A)$, folglich muss der Polarkegelschnitt der Geraden für das neue Büschel $(\mathfrak{A}, \mathfrak{B})$ durch die vorigen drei festen Punkte gehen w. z. b. w. *).

8. Wir kehren jetzt zu der in (4.) abgebrochenen Betrachtung zurück. Nehmen wir auf einer beliebigen Fläche zweiter Ordnung $F^{(2)}$ einen festen

*) Die Eigenschaften der Tripelcurve sind von Hrn. Hesse auf analytischem Wege abgeleitet worden. Vergl. den Aufsatz: Ueber Curven dritten Grades etc. in diesem Journal Bd. XXXVI, p. 143 etc. 
Punkt $O$ und denken uns in einer Ebene $E$ ein Kegelschnittnetz mit seiner Schaar-Schaar von Kegelschnitten gegeben, so wird jeder Kegelschnitt desselben in der Ebene $\boldsymbol{E}$ ein Polarsystem bestimmen, welches mit $\boldsymbol{O}$ verbunden ein Polarbündel giebt, und nach dem Satze (2.) liefert ein solches einen bestimmten Punkt $s$ im Raume. Wir fragen nach dem Ort derjenigen. Punkte $s$, welche såmmtlichen Kegelschnitten des Netzes entsprechen. Nach dem früheren Satze (3.) liegen solche Punkte $s$, welche den Kegelschnitten eines Büschels entsprechen, selbst auf einem Kegelschnitt $\mathfrak{K}$ und da die Kegelschnitte des Netzes sich in doppelt unendlicher Weise zu Büscheln zusammenfassen lassen (7.), so bilden die Punkte $s$ des gesuchten Ortes doppelt unendlich viele Kegelschnitte $\Omega$, die in gewisser Weise im Raume vertheilt sind. Um einen solchen Kegelschnitt $\mathfrak{R}$ zu erhalten, haben wir nach (3.) nur nöthig, die Tangentialebene an $F^{(2)}$ in $O$ zu-construiren, welche die Ebene $E$ in der Geraden $t$ schneide; dann wird der Polarkegelschnitt von $t$ in Bezug auf ein Büschel des Netzes mit $O$ verbunden einen Kègel liefern, welcher die Verbindungsebene der drei Schnittpunkte der $F^{(2)}$ mit den von $O$ nach dem gemeinschaftlichen Tripel des Büschels hingehenden drei Strahlen in dem gesuchten Kegelschnitte $\Re$ schneidet. Da nun nach (7.) die Polarkegelschnitte der Geraden $t$ in Bezug auf alle Büschel des Netzes durch drei feste Punkte $Q_{0} Q_{0}^{\prime} Q_{0}^{\prime \prime}$ gehen, so müssen alle Kegel, welche $O$ mit diesen Polarkegelschnitten verbinden, durch drei feste Strahlen $O Q_{1}, O Q_{0}^{\prime}, O Q_{0}^{\prime \prime}$ gehen, welche $g g^{\prime} g^{\prime \prime}$ heissen mögen, und da jeder Kegelschnitt $\Re$ des gesuchten Ortes auf einem solchen Kegel liegen muss, so treffen alle Kegelschnitte $\Re$ dieselbcn drei festen Geraden $g g^{\prime} g^{\prime \prime}$, welche in 0 zusammenlaufen.

Diese drei Geraden erscheinen selbst doppelt gezählt als drei specielle Kegelschnitte $\Omega$ des gesuchten Ortes (zusammenfallende Linienpaare), denn seien $\boldsymbol{P}_{0} \boldsymbol{P}_{0}^{\prime} \boldsymbol{P}_{0}^{\prime \prime}$ diejenigen drei Punkte, in welchen $\boldsymbol{t}$ der Tripelcurve des Netzes begegnet, so sind sie nach (5.) die conjugirten Punkte der Tripelcurve zu $Q_{0} Q_{0}^{\prime} Q_{0}^{\prime \prime}$ und es bilden diese sechs Punkte vier Tripel der Tripelcurve:

$$
Q_{0} Q_{1}^{\prime} Q_{0,}^{\prime \prime}, \quad Q_{0} P_{0}^{\prime} P_{0}^{\prime \prime}, \quad Q_{0}^{\prime} P_{0}^{\prime \prime} P_{0}, \quad Q_{0}^{\prime \prime} P_{0} P_{0}^{\prime} \text {. }
$$

Nehmen wir das Tripel $Q_{0} P_{0}^{\prime} P_{0}^{\prime \prime}$ verbinden es mit $O$ und suchen die drei Schnittpunkte dieser Strahlen mit $F^{(2)}$ auf, so fallen zwei derselben in $O$ hinein, weil $O P^{\prime} P^{\prime \prime}$ Tangentialebene an $F^{(2)}$ ist, der dritte aber ist der Schnittpunkt von $O Q_{0}$ oder $g$ mit $F^{(2)}$; die Verbindungsebene dieser drei Schnittpunkte wird also unbestimmt, muss aber durch $g$ gehen. Der Polarkegelschnitt der Geraden $t$ in Bezug auf das Büschel, dessen gemeinschaftliches Tripel $Q_{0} P_{0}^{\prime} P_{0}^{\prime \prime}$ 
ist, zerfällt in ein Linienpaar $Q_{0} P_{0}^{\prime}$ und $Q_{0} P_{0}^{\prime \prime}$, welches mit $O$ verbunden ein Ebenenpaar giebt; dieses Ebenenpaar geht selbst durch die Gerade $g$, hat also mit der vorhin aufgesuchten Ebene die Gerade $g$ doppelt gemein und diese ist daher als ein zusammenfallendes Linienpaar anzusehen, welches einen speciellen Kegelschnitt $\Re$ bildet, den Ort solcher Punkte $s$, welche allen Kegelschnitten des Büschels entsprechen, dessen gemeinschaftliches Tripel $Q_{0} P_{0}^{\prime} P_{0}^{\prime \prime}$ ist. Ebenso liefern die Kegelschnitte der beiden anderen Büschel, welche $\boldsymbol{Q}_{0}^{\prime} \boldsymbol{P}_{0}^{\prime \prime} \boldsymbol{P}_{0}$ und $\boldsymbol{Q}_{0}^{\prime \prime} \boldsymbol{P}_{0} \boldsymbol{P}_{0}^{\prime}$ zu gemeinschaftlichen Tripeln haben, als Ort der ihnen entsprechenden Punkte $s$ die doppelt zu nehmenden Geraden $g^{\prime}$ und $g^{\prime \prime}$.

Die Ebene eines Kegelschnitts $\Re$ enthält immer noch einen zweiten Kegelschnitt $\Omega^{\prime}$, der ebenfalls dem gesuchten Orte angehört; denn die drei von $O$ nach irgend einem Tripel der Tripelcurve gezogenen Strahlen treffen $F^{(2)}$ in drei Punkten, deren Verbindungsebene $F$ den drei festen Strahlen $g g^{\prime} g^{\prime \prime}$ in drei solchen Punkten begegnet, dass diese sechs Punkte auf einem Kegelschnitte $\mathfrak{R}$ liegen, die Ebene $\mathfrak{F}$ schneidet aber $F^{(2)}$ in einem Kegelschnitt $K$, und der von $O$ durch $K$ gelegte Kegel trifft die Tripelcurve ausser in den ersten drei Tripelpunkten noch in drei neuen Punkten, welche (7.) ein zweites Tripel der Tripelcurve sind; die Strahlen von $O$ nach den letzteren gezogen treffen die Fläche $F^{(2)}$ in drei Punkten, welche auf $\boldsymbol{K}$ liegen, deren Verbindungsebene also dieselbe Ebene $\Subset$ ist und diese Punkte müssen mit den vorigen Schnittpunkten von $₹$ und den Strahlen $g g^{\prime} g^{\prime \prime}$ in einem zweiten Kegelschnitt $\mathfrak{\Re}^{\prime}$ liegen, der offenhar zum gesuchten Orte gehört. Die beiden Kegelschnitte $\Re$ und $\mathfrak{K}^{\prime}$, welche in derselben Ebene liegen, schneiden sich ausser in den drei auf $g g^{\prime} g^{\prime \prime}$ befindlichen Punkten noch in einem vierten Punkte; dieser entspricht dem einzigen Kegelschnitt, welcher beiden Büscheln des Netzes gemeinschaftlich ist (7.), die $\Re$ und $\mathfrak{\Omega}^{\prime}$ liefern.

Es ist ferner ersichtlich, dass alle Punkte, in welchen der von $O$ durch die Tripelcurve gelegte Kegel dritten Grades der $\boldsymbol{F}^{(2)}$ begegnet, die also auf einer Raumcurve sechsten Grades liegen, dem gesuchten Orte angehören müssen, weil die Tripelcurve der Ort der gemeinschaftlichen Tripel für alle Büschel des Netzes ist. Die Punkte $Q_{0} Q_{0}^{\prime} Q_{0}^{\prime \prime}$ bilden ein besonderes Tripel, dessen zugehöriges Büschel einen Kegelschnitt $\mathfrak{R}_{0}$ liefert, der durch diejenigen Punkte geht, in welchen $g g^{\prime} g^{\prime \prime}$ die $F^{(2)}$ treffen. Die Ebene dieses Kegelschnittes schneidet $F^{(2)}$ in einem Kegelschnitte $K_{0}$ und der von 0 durch $\boldsymbol{K}_{0}$ gelegte Kegel trifft die Tripelcurve in drei neuen Punkten, die auch ein Tripel der Tripelcurve sind. Das diesem Tripel zugehörige Büschel des Netzes 
liefert aber einen Kegelschnitt $\mathfrak{\Omega}_{v}^{\prime}$, der offenbar mit $K_{0}$ zusammenfällt; folglich enthält der gesammte Ort der Punkte $s$ 1) in doppelt unendlichvielen Ebenen Kegelschnittpaare $\mathfrak{R}$ und $\mathfrak{R}^{\prime}$ 2) drei bestimmte doppelt zu nehmende Gerade gg' $^{\prime \prime}{ }^{\prime \prime}$, welche in einem Punkte $O$ zusammenlaufen und auf denen sich je zwei Kegelschnitte $\mathfrak{\Re}$ und $\mathfrak{\Omega}^{\prime}$ treffen 3) auf der Fläche $F^{(2)}$ eine bestimmte Raumcurve sechsten Grades, die Durchschnittscurve mit demjenigen Kegel dritten Grades, welcher $\mathrm{O}$ mit der Tripelcurve des Netzes verbindet, und noch einen besonderen Kegelschnitt $K_{0}$, welchen die Ebene ausschneidet, die durch die drei Schnittpunkte der Doppelpunktlinien $g^{\prime} g^{\prime \prime}$ mit $F^{(2)}$ gelegt wird.

9. Da sich die Schaar-Schaar sämmtlicher Kegelschnitte des Netzes in der Weise (6.) zusammenfassen lässt, dass wir von drei beliebigen Kegelschnitten $A, B, C$, die nicht demselben Büschel angehören, ausgehen, einen veränderlichen Kegelschnitt $\mathfrak{A}$ des Büschels $(B, C)$ mit $A$ zu einem Büschel $(A, \mathfrak{A})$ zusammenstellen und dann die Kegelschnitte sämmtlicher Büschel $(A, \mathfrak{A})$ als die Schaar-Schaar Kegelschnitte des Netzes erhalten, so wird auch der ganze Ort der Punkte $s$ als eine continuirliche Reihe von Kegelschnitten $\Re$ im Raume angesehen werden können, die den Büscheln $(A, \mathfrak{A})$ entsprechen. Diese $\mathfrak{R}$ gehen sämmtlich durch einen Punkt $s_{a}$, der dem Kegelschnitte $A$ entspricht, und durch eine Reihe von Punkten $s_{\mathfrak{a}}$, die auf einem bestimmten Kegelschnitte $\mathfrak{\Omega}_{(B, C)}$ liegen, dessen Punkte den sämmtlichen Kegelschnitten $\mathfrak{A}$ des Büschels $(B, C)$ entsprechen. Die Ebenen der $\mathfrak{K}$ umhüllen also einen gewissen Kegel, den wir ermitteln wollen; jede solche Ebene enthält unmittelbar nur einen Kegelschnitt $\mathfrak{R}$ des Ortes, aber die Kegelschnitte der andern Ebenen begegnen ihr in Punktenpaaren, die ebenfalls zum Orte gehören. Da wir von jedem dieser Kegelschnitte $\mathfrak{R}$ vorerst nur zwei Punkte $s_{a}$ und $s_{\mathfrak{a}}$ kennen, so müssen wir zur Bestimmung seiner Ebene noch einen dritten Punkt zu ermitteln suchen; dies gelingt in folgender Weise: Denken wir uns aus dem Büschel $(C, A)$ des Netzes einen beliebigen Kegelschnitt $\mathfrak{B}$ gewählt, so muss nach dem oben bewiesenen Satze (5.), weil $C, B, \mathfrak{A}$ einem Büschel und $C, A, \mathfrak{B}$ einem zweiten Büschel angehören, welches mit dem ersten den Kegelschnitt $C$ gemein hat, den beiden Büscheln $(A, \mathfrak{A})$ und $(B, \mathfrak{B})$ ein einziger bestimmter Kegelschnitt gemeinschaftlich sein, welcher $\mathfrak{A}^{\prime}$ heisse. Es verändert sich mit $\mathfrak{A}$ auch $\mathfrak{A}^{\prime}$, während beide respective den Büscheln $(B, C)$ und $(B, \mathfrak{B})$ angehören und zwei zusammengehörige $\mathfrak{A}^{\prime}$ schneiden sich in je vier Punkten auf dem Kegelschnitte $A$. Fällt insbesondere $\mathfrak{A}$ mit $B$ zusammen, so fällt auch $\mathfrak{A}^{\prime}$ mit $B$ zusammen. Wir haben mithin die Kegelschnitte $\mathfrak{A}^{\prime} \mathfrak{\prime}^{\prime}$ zweier 
Büschel $(B, C)$ und $(B, \mathfrak{B})$ so auf einander bezogen, dass zwei entsprechende in dem gemeinschaftlichen Kegelschnitte $B$ zusammenfallen und alle übrigen Paare entsprechender Kegelschnitte sich in je vier Punkten eines festen Kegelschnitts $A$ schneiden; hieraus folgt (als specieller Fall des Erzeugnisses zweier projectivischen Kegelschnittbüschel, welches eine Curve vierten Grades ist, die hier in zwei Kegelschnitte zerfällt), dass die beiden Büschel, welche $\mathfrak{A}$ und $\mathfrak{A}^{\prime}$ durchlaufen, in projectivischer Beziehung stehen, dass also auch die beiden von den Punkten $s_{\mathfrak{a}}$ und $s_{\mathfrak{a}}$ durchlaufenen krummen Punktreihen auf den Kegelschnitten $\mathfrak{R}_{(B, C)}$ und $\mathfrak{R}_{(B, \mathfrak{B})}$, die jenen Büscheln hinsichtlich $F^{(2)}$ zugehören, projectivisch sein und einen Punkt $s_{b}$ gemeinschaftlich haben müssen, in welchem gleichzeitig zwei entsprechende Punkte der krummen Punktreihen zusammenfallen. Hieraus ergiebt sich, dass die durch den unveränderlichen Punkt $s_{a}$ und je zwei entsprechende Punkte $s_{\mathfrak{a}}$ und $s_{\mathfrak{a}^{\prime}}$ gelegte Ebene einen Kegel dritter Classe mit einer Doppeltangentialebene also nur vierten Grades umhüllen wird*). Der Ort der Ebenen aller in obiger Weise gruppirten Kegelschnitte $\Re$ ist daher dieser Kegel dritter Klasse mit einer Doppeltangentialebene; die letztere enthält zwei von den betrachteten Kegelschnitten $\Omega$ und $\Omega^{\prime}$, jede andere Tangentialebene nur einen. Denken wir uns nun durch den Punkt $s_{a}$, an dessen Stelle jeder andere Punkt des gesuchten Ortes treten kann, eine beliebige Gerade $G$ gezogen, so gehen durch dieselbe im Allgemeinen drei Tangentialebenen des so eben ermittelten Kegels und jede derselben enthält einen bestimmten Kegelschnitt $\Re$, der durch $s_{a}$ geht; folglich enthält die Gerade $G$ von dem gesammten Ort der Punkte $s$ höchstens vier, den Punkt $s_{a}$ und die drei anderen Schnittpunkte der drei durch $s_{a}$ gehenden soeben ermittelten Kegelschnitte. Da aber $s_{a}$ ganz willkührlich gewählt ist, so schliessen wir, dass jede beliebige Gerade im Allgemeinen vier Punkte $s$ des gesuchten Ortes enthält, dass also der Ort sämmtlicher Punkte $s$ eine Flache vierten Grades bildet. Die Punktenpaare, in welchen die Ebene eines bestimmten Kegelschnitts $\mathfrak{R}$ von allen übrigen Kegelschnitten $\mathfrak{\Omega}$ getroffen wird, müssen daher einen zweiten Kegelschnitt $\mathfrak{K}^{\prime}$ bilden, wie wir dies schon früher erkannt haben. Eine solche Ebene, welche die Fläche vierten Grades in zwei Kegelschnitten $\Omega$ und $\AA^{\prime}$ schneidet, ist als Tangentialebene derselben anzusehen; von den vier Schnittpunkten der Kegelschnitte $\Re$ und $\Re^{\prime}$ sind nämlich drei die auf den Doppelkanten $g g^{\prime} g^{\prime \prime}$ der Fläche

*) Siehe dieses Journal Bd. 54, Seite $31 \mathrm{ff}$. 
liegenden wirklichen Doppelpunkte (8.), der vierte muss aber Berührungspunkt der Ebene mit der Fläche sein, weil von ihm aus nach zwei verschiedenen Richtungen zwei unendlich nahe Punkte der Fläche (auf $\mathfrak{R}$ und $\mathfrak{\Omega}^{\prime}$ ) zugleich in der Ebene liegen; da die durch den ganz willkührlichen Punkt $s_{a}$ der Fläche vierten Grades gehende Doppeltangentialebene des vorhin ermittelten Kegels dritter Klasse zwei Kegelschnitte $\Re$ und $\Re^{\prime}$ enthält, die durch $s_{a}$ gehen, so ist sie Berührungsebene der Steinerschen Fläche in diesem Punkte und wir können schliessen, dass jede Berührungsebene der Steinerschen Fläche dieselbe in einem Kegelschnittpaar schneidet. Hieraus lässt sich auch ermitteln, von der wievielsten Classe die Steinersche Fläche vierten Grades ist; denn treffe sie eine beliebige Gerade $G$ in den vier Punkten $\alpha \beta \gamma \delta$, so gehen nach dem Vorigen durch den Punkt $\alpha$ und die Gerade $G$ im Allgemeinen drei Ebenen $E_{1} E_{2} E_{3}$, welche drei Kegelschnitte $\Re$ enthalten; diese müssen in den Punkten $\alpha \beta, \alpha \gamma, \alpha \delta$ die Gerade $G$ treffen; jede der Ebenen enthält aber noch einen zweiten Kegelschnitt $\AA^{\prime}$ und diese müssen, weil es nur vier Punkte $s$ auf der Geraden $G$ giebt, respective durch $\gamma \delta, \beta \delta, \beta \gamma$ gehen; die drei Kegelschnittpaare in den Ebenen $E_{1} E_{2} E_{3}$ schneiden also die Gerade $G$ in folgender Weise: das Kegelschnittpaar in $E_{1}$ trifft $G$ in den Punktenpaaren $\alpha \beta$ und $\gamma \delta$

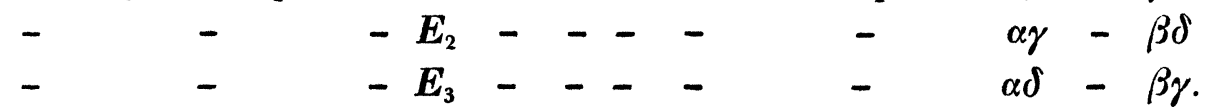

Durch den Punkt $\beta$ und die Gerade $G$ giebt es nun auch nur drei Ebenen, welche drei Kegelschnittpaare enthalten, die in den Punkten $\beta \gamma$ und $\delta \alpha, \beta \delta$ und $\alpha \gamma, \beta \alpha$ und $\gamma \delta$ der Geraden $G$ begegnen müssen; da aber die vorigen Ebenen diese Eigenschaft besitzen, so sind sie mit den neuen identisch; es giebt daher durch eine beliebige Gerade $G$ im Allgemeinen nur drei Ebenen, welche Kegelschnittpaare aus der Steinerschen Fläche ausschneiden, also dieselbe berühren; die Steinersche Fläche vierten Grades ist also nur dritter Classe. Hieraus folgt auch, dass es durch einen beliebigen Punkt im Raume unendlich viele Ebenen giebt, welche Kegelschnittpaare aus der Steinerschen Fläche ausschneiden und dass dieselben einen Kegel dritter Classe also im Allgemeinen sechsten Grades umhüllen; dieser ist aber nur vom vierten Grade, weil er eine Doppeltangentialebene enthält, sobald der angenommene Punkt anf der Fläche selbst liegt, und er degenerirt in einen Kegel zweiter Classe, also auch zweiten Grades, sobald der Punkt auf einer der drei Doppellinien $g g^{\prime} g^{\prime \prime}$ liegt; was denn auch mit den von Herrn Kummer a. a. 0. mitgetheilten Resultaten übereinkommt. 